\title{
Biomimetic synthesis of hybrid nanocomposite scaffolds by freeze-thawing and freeze-drying
}

\author{
S NAYAR*, A K PRAMANICK, A GUHA, B K MAHATO, M GUNJAN and A SINHA \\ National Metallurgical Laboratory, Jamshedpur 831 007, India
}

\begin{abstract}
The aim of this study is to biomimetically synthesize hydroxyapatite-hydrophilic polymer scaffolds for biomedical applications. This organic-inorganic hybrid has been structurally characterized and reveals a good microstructural control as seen by the SEM analysis and the nanosize of the particulates is confirmed by AFM microscopy. The characterization of such nano-structured composites would allow researchers to design new systems, tailoring properties for different applications.
\end{abstract}

Keywords. Biomimetics; nanocomposites; hydroxyapatite; scaffolds; hydrophilic polymers.

\section{Introduction}

One of the most important issues in the synthesis of nanoparticles is knowing how to regulate the particle size and morphology. Biomimetics, a concept derived from Mother Nature and implemented in the design and synthesis of nanoscale materials, has been successful in creating functional nanomaterials and structures (Sinha et al 2001). Biomimetic synthesis realizes an in situ nucleation and growth of inorganic nanoparticles in a pre-organized organic matrix (Mann and Ozin 1996). The microstructural sophistication thus achieved by interfacial interactions provides much better functional properties than mere polymer-inorganic blends. It is established that different hydrophilic polymers can be used as pre-organized matrices in the biomimetic synthesis of a wide range of nanosystems including polymer-inorganic nanocomposites for tissue engineering (Sinha et al 2003). Here, we have used poly (vinyl) alcohol (PVA). Owing to its chemical composition very similar to the major component of human bone, hydroxyapatite (HAp) reinforced polymer nanocomposites are increasingly used as implants for medical applications (Nayar and Sinha 2004; Dorozhkin 2007). In situ synthesis of HAp in PVA not only overcomes the poor mechanical properties of HAp but also provides a range of possibilities to create different forms of hybrid scaffolds for bone and cartilage grafts (Sinha et al 2007). The molecular recognition in these hybrid materials not only controls the shape and dimensions of HAp nanoparticles, yet assembles them into higher dimensional structures. In this paper, we describe how after the same initial biomimetic-synthesis of HAp in PVA, two different postsynthesis processing techniques lead to two different scaffold forms. A two-dimensional flat sheet after freeze-

\footnotetext{
*Author for correspondence (nayar@nmlindia.org)
}

drying and a three-dimensional block after repeated cycles of freeze thawing are formed. Both scaffolds were characterized by X-ray diffractometry (XRD), scanning electron microscopy (SEM) and atomic force microscopy (AFM).

\section{Experimental}

Biomimetic synthesis of HAp in PVA was established in our laboratory and the process patented by our group (Sinha et al 2005). In order to get 2-D fabric and $3-D$ block forms, we integrated this process with low temperature phase separation, achieved by freeze-drying and freeze-thawing, the patent of which is under way. In lyophilization, the temperature was drastically dropped to about $-80^{\circ} \mathrm{C}$ whereas in freeze-thawing there were repeated cycles of freezing-thawing wherein water was entrapped and retained in the system. The chemicals were obtained from the following sources: PVA was from Qualigens with a molecular weight, $1,25000, \mathrm{Ca}\left(\mathrm{NO}_{3}\right)_{2}$ tetrahydrate from Hi Media and $\left(\mathrm{NH}_{4}\right)_{2} \mathrm{HPO}_{4}$ from Merck. While in freeze drying, the final slurry was dried at $-80^{\circ} \mathrm{C}$ at a very fine vacuum of 0.03 milli bar for $36-48 \mathrm{~h}$ and packed and in freeze-thawing the synthesized slurry was kept in the freezer $\left(4^{\circ} \mathrm{C}\right)$ of a frost free refrigerator. It was frozen and then thawed at room temperature. This thermal cycling was repeated on an average about 15 times whereby a nice hydrogel was obtained.

\section{Results and discussion}

Bone is a hierarchical nanocomposite built from ceramic tablets and organic binders, and is therefore, a subject of immense interest for materials scientists. PVA is a wellknown hydrophilic biocompatible polymer and here we have capitalized on its ability to in situ synthesize HAp 

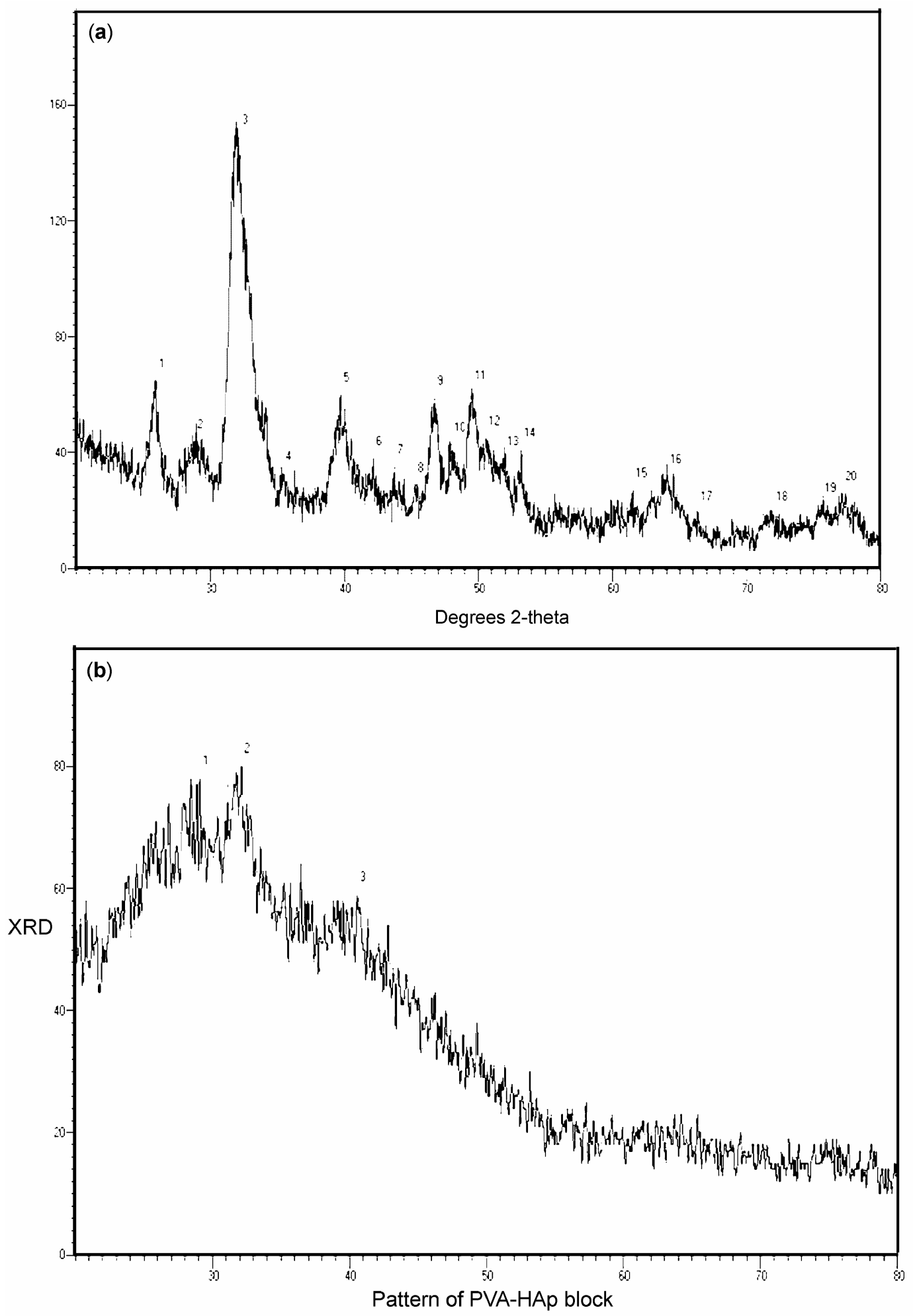

Figure 1. XRD patterns of (a) PVA-HAp sheet and (b) PVA-HAp block.

and physically cross-link itself under subjected conditions. The XRD studies of both the scaffolds (the sheet and the block) forms revealed HAp as the predominant phase with the following characteristic peaks (210), (112),
(310), (221) and (113) for bandage and (210), (112) and (221) peaks for block (figure 1). An appreciable degree of crystallinity present in the PVA-HAp nanocomposites is attributed to the surface energy of the organic surface which 
acts as a nucleation catalyst, however, the growth remains limited due to sluggish diffusion process in polymer matrix. The block XRD data shows less crystallinity because of the entrapped water molecules. The SEM micrographs clearly reveal that both the scaffolds are made up of nonwoven structures of cross-linked PVA-HAp nanofibrils having diameter in the range of 100-150 $\mathrm{nm}$ (figure 2). TEM analysis of the sample confirmed that PVA nanofibrils are highly mineralized with HAp nanoparticles (figure 3a). The sheet like nanocomposite revealed the HAp particle size to be about $\sim 10 \mathrm{~nm}$, however, a relatively larger particle size $(80-100 \mathrm{~nm})$ is obtained in PVA-HAp block (figure $3 \mathrm{~b}$ ). This seems to be logical as prolonged thermal cycling of biomimetic PVA-HAp provides ample time for the diffusion necessary for the growth of HAp particles in PVA matrix, hence, the size of the particles appears bigger.

\subsection{AFM studies in non-contact mode}

The topographic images reveal the particle-tip interaction and not the morphology. In compliance with the TEM data, the AFM images reveal an apparent clustering or
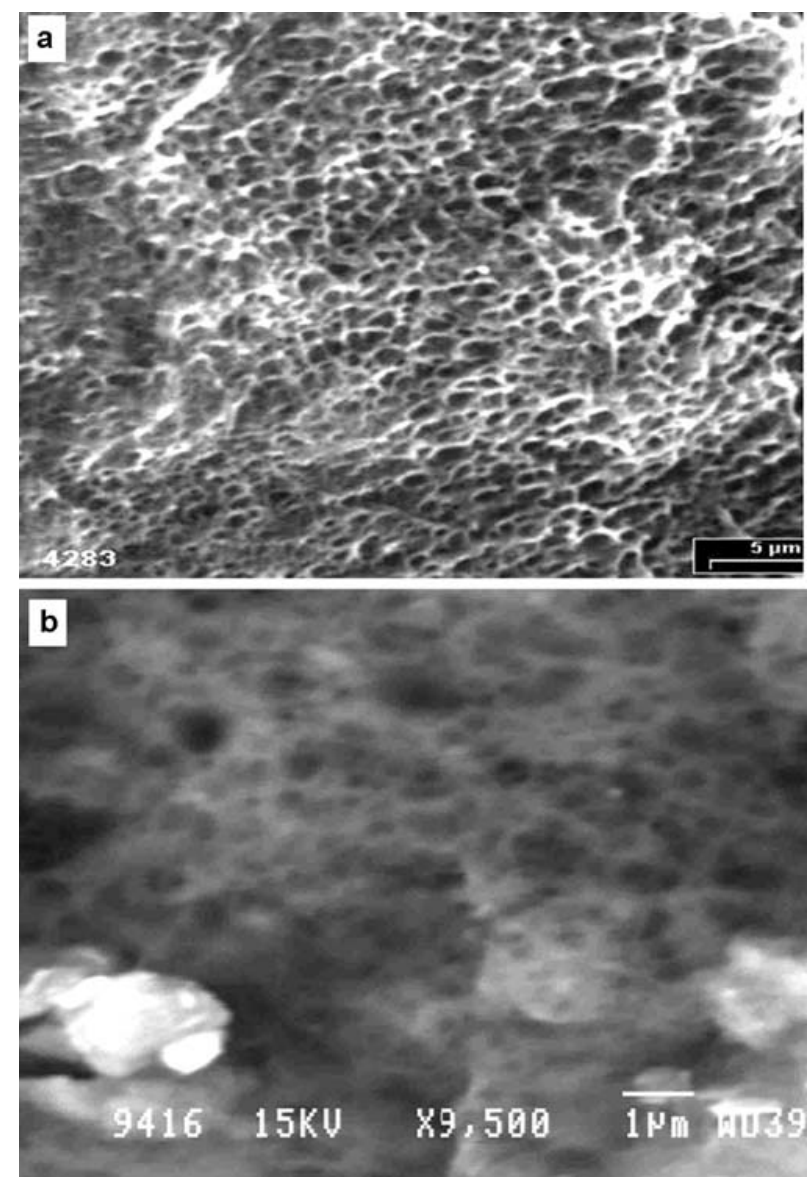

Figure 2. SEM micrographs of a. PVA-HAp nanocomposite sheet and $\mathbf{b}$. PVA-HAp nanocomposite block. growth in the freeze-thawed sample in comparison with the lyophilized one. It also reflects the narrow size distribution of the synthesized HAp particles (figures $4 a$ and $b$ ).

Scaffolds used in tissue engineering mimic the natural extracellular matrix and provide support for cell adhesion, migration and proliferation. Though our initial biocompatibility tests before the post-synthesis processing are as per standard norms, we are in the process of making these scaffolds in bulk in order to get the tissue specific cell-adhesion tests done.

\section{Conclusions}

The results shown here provide an excellent illustration of the symbiotic relationship that nanotechnology shares with innate polymer chemistry whether it is synthetic or biopolymers. We have initiated scaffold synthesis using HAp, PVA and collagen in both the sheet and block and the results are very encouraging. In polymeric systems, nanostructures play the role of nonreactor for growing nanoparticles. These materials are not only simple and cheap; they even control particles growth. Though our
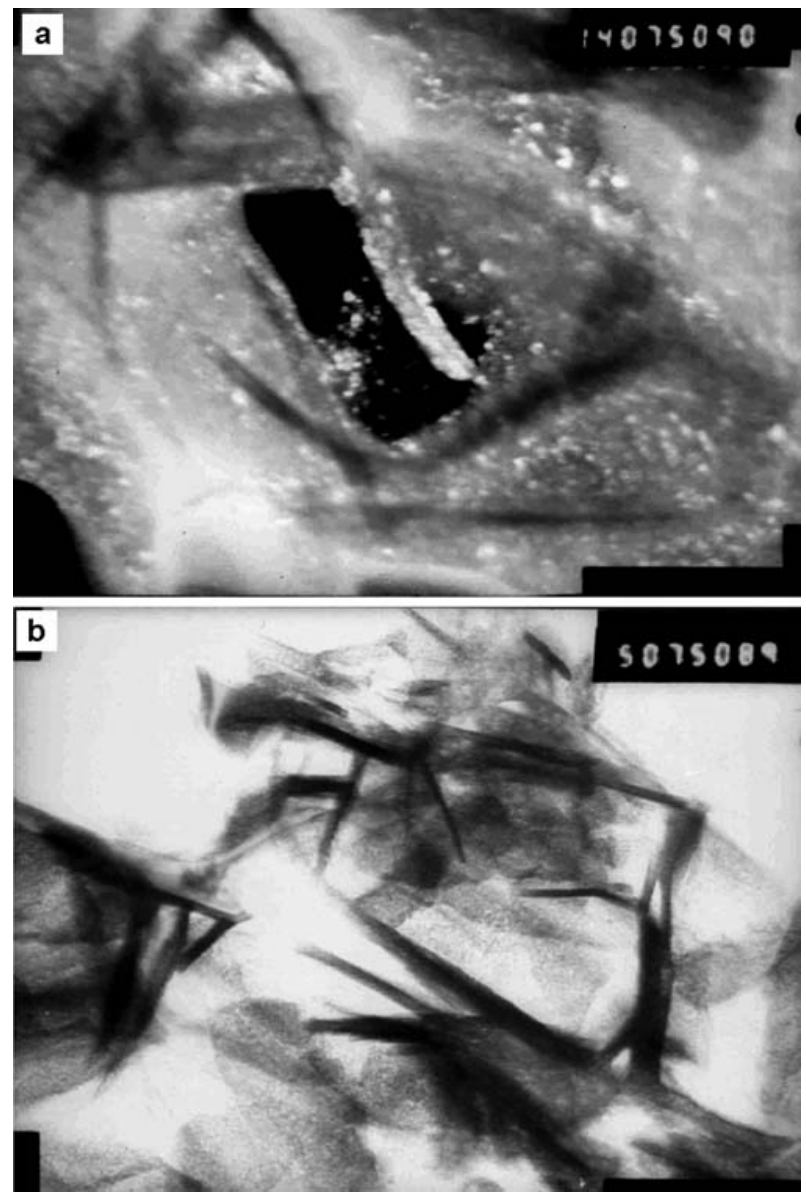

Figure 3. TEM images revealing precipitation of HAp nanoparticles in a. PVA sheet and $\mathbf{b}$. PVA block. 

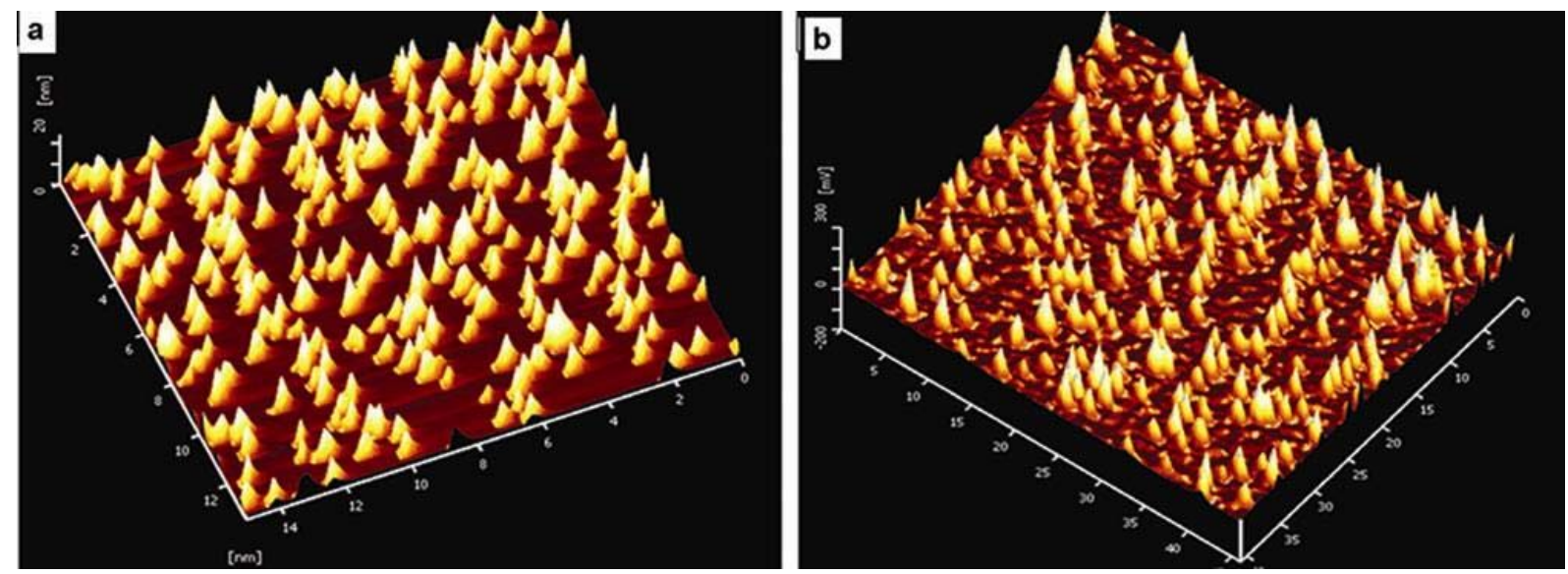

Figure 4. AFM topography revealing nanosized HAp particles in a. PVA sheet and b. PVA block.

results show improved microstructural and mechanical properties, for ideal tissue engineering, the desired tissue should be used as the model to engineer the chemical, mechanical and biological properties into the scaffold.

\section{References}

Dorozhkin S V 2007 J. Mater. Sci. 421061

Mann S and Ozin G A 1996 Nature 382313

Nayar S and Sinha A 2004 Colloid \& Surface B35 29
Sinha A, Das S K, Rao V and Ramachandrarao P $2001 \mathrm{~J}$. Mater. Res. 461846

Sinha A, Nayar S, Agrawal A, Bhattacharya D and Ramachandrarao P 2003 J. Am. Ceram. Soc. 86357

Sinha A, Nayar S, Agrawal A, Rao V and Ramachandra Rao P 2005 Indian Patent 'A process for preparation of nanosize hydroxyapatite by a biomimetic route', Patent Number 192392

Sinha A, Das G, Sharma B K, Roy R P, Pramanik A K and Nayar S 2007 Mater. Sci. Engg. C27 70 